\title{
Non-Recursive Adaptive Control Design Methodology for Nonlinear Systems With Mismatched Time-Varying Disturbances
}

\section{Xin Dong}

Shanghai University of Electric Power

Zhang Chuanlin ( $\sim$ clzhang@shiep.edu.cn )

Shanghai University of Electric Power https://orcid.org/0000-0001-6052-1682

Chenggang Cui

Shanghai University of Electric Power

\section{Research Article}

Keywords: adaption mechanism, mismatched disturbances, higher-order sliding mode observer, lowertriangular nonlinear system

Posted Date: March 10th, 2021

DOI: https://doi.org/10.21203/rs.3.rs-232694/v1

License: (9) This work is licensed under a Creative Commons Attribution 4.0 International License. Read Full License 


\title{
Non-recursive adaptive control design methodology for nonlinear systems with mismatched time-varying disturbances
}

\author{
Xin Dong - Chuanlin Zhang - Chenggang Cui
}

Received: date / Accepted: date

\begin{abstract}
This paper proposes a non-recursive adaptive control scheme for a class of lower-triangular nonlinear systems with mismatched time-varying disturbances. As a result, an exact tracking control scheme is constructed straightforwardly from the system in a novel non-recursive synthesis manner. Firstly, with the help of higher-order sliding mode observer (HOSMO), the original system is delicately transformed into an equivalent stabilizable system. Then, a nonrecursive stabilizer with a simple update mechanism for the dynamic gain can be derived. Subsequently, a rigorous stability analysis shows the theoretical justification of the proposed design framework. New characteristics of the proposed algorithm are mainly twofold: 1) The proposed adaption mechanism could substantially adjust the transient-time performance with the presence of different levels of disturbances. 2) The composite control design procedure is essentially detached with stability analysis, which could largely facilitate practical implementations.
\end{abstract}

Keywords adaption mechanism $\cdot$ mismatched disturbances $\cdot$ higher-order sliding mode observer $\cdot$ lowertriangular nonlinear system

Xin Dong · Chuanlin Zhang · Chenggang Cui

Shanghai Engineering Research Center of Intelligent Management and Control for Power Process, College of Automation Engineering, Shanghai University of Electric Power, Shanghai 200090, China

email: dongxin@mail.shiep.edu.cn

Chuanlin Zhang

email: clzhang@shiep.edu.cn

Chenggang Cui

email: cgcui@shiep.edu.cn

\section{Introduction}

In this paper, we revisit a class of lower-triangular nonlinear systems with mismatched time-varying disturbances, which can be depicted as the following form

$\left\{\begin{array}{l}\dot{x}_{i}(t)=x_{i+1}(t)+f_{i}\left(\bar{x}_{i}(t)\right)+d_{i}(t), i \in \mathbb{N}_{1: n-1}, \\ \dot{x}_{n}(t)=u(t)+f_{n}(x(t))+d_{n}(t), \\ y(t)=x_{1}(t)\end{array}\right.$

where $\bar{x}_{i}=\operatorname{col}\left(x_{1}, x_{2}, \cdots, x_{i}\right), i \in \mathbb{N}_{1: n}, x=\bar{x}_{n}, u$ and $y$ are the system partial state vectors, full state vector, control input and output respectively. $f_{i}(\cdot), i \in \mathbb{N}_{1: n}$ is a known nonlinear function which satisfies the Lipschitz continuous condition. $d_{i} \in \mathcal{L}_{\infty} \cap C^{n-i}$ depicts the mismatched disturbance term. Throughout this paper, the initial time is set as $t_{0}=0$, the initial state vector is denoted by $x_{0} \triangleq x(0)$ and the reference output tracking trajectory is denoted by $y_{r}$.

Constructing an exact tracking control strategy for nonlinear systems including system (1) with high-performance is always a challenging but significant task for control engineering industries. Owing to the blossom of disturbance measurement and identification tools over the past two decades, plenty of tracking control methods for uncertain nonlinear systems have been developed. Notably, by incorporating various disturbance observation strategies, a category of disturbance/uncertainty estimation and attenuation (DUEA) control algorithms has aroused continuous attention from control community [1]. For instance, in order to enhance system anti-disturbance ability and robustness against uncertain coefficients, a nonlinear disturbance observer based control (DOBC) approach is well established in [2]. An alternative active disturbance rejection control (ADRC) methodology is reported in [3]. Meanwhile, an extended high-gain observer can also be found in [4], which could recover the performance of the nominal linear model as observer gains 
are sufficiently large. A practical finite-time disturbance decoupling result is presented and a practical finite-time tracking controller is designed by means of prescribed performance control in [5]. Based on the above pioneer results, there are many extensions and further results regarding DUEA can be widely found in both theoretical and applicational fields later on, see e.gs., [6]-[11], etc.

It is worthy of pointing out here that for a class of nonlinear systems perturbed by mismatched disturbances/uncertainties, rather than conventional matched case, fewer results can be referred to in the literature. One main difficulty relies on the fact that control input channels are different from the mismatched disturbances, and it is generally impossible to transfer the mismatched disturbances to the same channel as the control inputs by coordinate transformation (see, for instance, [1], p. 95).

Aiming to obtain accurate estimation of mismatched disturbances, an interesting result is presented in [12], where an extended state observer (ESO) with less dependence of the system information is developed, which enables an easytransplantable characteristic for nonlinear systems with variable nonlinearity restrictions. Later on, the work [13] develops a sliding mode controller for systems with unmatched uncertainties by nonlinear disturbance observation. For a class of nonlinear systems of the form (1), constructing a series of virtual controllers via backstepping approach is a common idea to handle the tracking control problem. Combined with a non-linear disturbance observer, a recursive result is obtained in [14], which introduces the disturbance estimation into virtual control law in each step to compensate the mismatched disturbances. A composite controller is proposed by integrating backstepping method with a HOSMO in order to handle unknown disturbances in [15], which can recover system performance within a finite time. However, recursive design commonly results in the complexity of controller design, especially for those systems with high order. Thereafter, the derived control schemes are usually totally hard to be implemented into practices. Aiming to ease the complexity of controller form, series of non-recursive control strategies are developed recently in the literature. Combining disturbance observation with homogeneous system theory, [16] proposes a novel non-recursive control framework, which could obtain an alternative methodology to realize exact trajectory tracking for system (1). However, it may lead to robustness redundancy of the closed-loop system, as the control gains tend to be chosen as sufficiently large in order to maintain the system stability under various working conditions. Hence, a natural conjecture arises here: "For system (1) with different levels of time-varying disturbances, can we derive an adaptive output regulation law under the non-recursive design framework in order to improve the dynamic control performance?"
As noted in the literature, in the case when system (1) satisfies $d_{i}(t)=0$ and $f_{i}$ is integrated with non-parametric uncertainties, a time-varying scaling gain method is presented in [17], which could derive a universal control law via dynamic high-gain domination technique. Inspired by this result, we present an adaptive tracking control scheme for system (1) in a novel non-recursive manner. To this aim, firstly, a higher-order sliding mode observer is employed to restore the performance of the perturbed system within a finite time. Secondly, we put forward a systematic feedforward framework to transform the original system into a stabilizable system form. Thirdly, by introducing a dynamic gain, the stability analysis could be greatly simplified and a composite controller law are constructed subsequently, which includes a linear feedback control law and a dynamic gain update law. At last, a rigourous system stability analysis is given to ensure the theoretical justification of the proposed design strategy.

Owing to the fact that the proposed controller is effectively detached with the stability analysis based on Lyapunov function, it could be straightforwardly derived from system with reduced calculation burdens.

In reference to existing related results, the main contributions of this paper are summarized by the following two aspects:

- With the presence of a variety of general mismatched disturbances, the proposed control scheme is not only able to adaptively adjust the transient-time performance, but also guarantee an offset-free tracking control result.

- Unlike popular recursive controller design methods, a non-recursive manner is adopted in the proposed controller design framework, which significantly reduces the complexity of controller construction.

The remainder of this paper is organized as follows. Section 2 introduces some preliminaries and problem statements. The main design framework with rigorous stability analysis is presented in Section 3. Section 4 provides a real-life simulation to demonstrate the effectiveness of the proposed method. The conclusion of this paper is placed in Section 5.

\section{Preliminaries and Problem Statements}

\subsection{Definitions and Lemma}

The following definitions and notations are provided for briefness of expressions.

- The symbols $\mathbb{N}, \mathbb{R}$ and $\mathbb{R}_{+}$denote the set of positive integers, real numbers and positive real numbers respectively. The symbol $C^{i}(i \in \mathbb{N})$ denotes the set of all differentiable functions whose first $i$-th time derivatives are continuous. The symbol $\mathcal{L}_{\infty}$ represents the set of all 
signals whose infinity-norms are bounded. The symbol $\mathbb{N}_{j: i} \triangleq\{j, j+1, \cdots, i\}, \forall i, j \in \mathbb{N}$ and $j \leq i$.

- $\forall P \in \mathbb{R}^{n \times n}, \lambda_{\max }(P)$ and $\lambda_{\min }(P)$ denote the maximum and the minimum eigenvalues of $P .\lfloor w\rceil^{r} \triangleq \operatorname{sign}(w)|w|^{r}, r$ $\in \mathbb{R}_{+}$.

Lemma 1 (Young's Inequality): If $p>1, q>1, \frac{1}{p}+\frac{1}{q}=1$, then $\forall a, b \geq 0$, the following inequality holds: $a \cdot b \leq \frac{a^{p}}{p}+\frac{b^{q}}{q}$.

\subsection{Problem Statements}

The following assumptions are made in priori.

Assumption 2.1 The reference signal $y_{r}$ and its $n$-th order derivative are piecewise continuous, known and bounded. Assumption 2.2 The mismatched disturbance $d_{i}(t)$ satisfies $\max _{i \in \mathbb{N}_{1: n}, j \in \mathbb{N}_{0: n-i+1}}\left\{\sup _{t \geq 0}\left|\frac{\partial d_{i}^{j}(t)}{\partial t^{j}}\right|\right\} \leq N$ with $N \in \mathbb{R}_{+}$being a constant.

In what follows, a linear control law and adaptive update law of this paper can be first designed as the following nonrecursive form:

$\left\{\begin{array}{l}u=u\left(K, x, x^{*}\right), \\ \dot{L}=\psi\left(L, x, x^{*}\right), L_{0}=1,\end{array}\right.$

such that an asymptotical stability can be deduced for resulting closed-loop system (1)-(2) as described in hereinafter.

\section{Main Results}

In this section, a step-by-step controller construction procedure is presented in an explicit manner.

\subsection{System Performance Recovery and Pre-treatment}

Firstly, assume that the disturbance vector $d=\operatorname{col}\left(d_{1} \cdots d_{1}^{(n-1)}\right.$, $d_{2} \cdots d_{2}^{(n-2)} \cdots d_{n}$ ) is known for system (1) and the output reference tracking trajectory is given as $y_{r}$. Then define an auxiliary state variable as $\bar{\varsigma}_{i}=\operatorname{col}\left(\varsigma_{1}, \varsigma_{2}, \cdots, \varsigma_{i}\right), i \in \mathbb{N}_{1: n+1}$, where $\varsigma_{i}$ is determined by the following output regulation equations

$\left\{\begin{array}{l}\varsigma_{1}\left(y_{r}\right)=y_{r}, \\ \varsigma_{i+1}\left(d, \bar{y}_{r}\right)=\dot{\varsigma}_{i}-f_{i}\left(\bar{\varsigma}_{i}\right)-d_{i}, i \in \mathbb{N}_{1: n},\end{array}\right.$

where $\bar{y}_{r}=\operatorname{col}\left(y_{r}, y_{r}^{(1)}, \cdots, y_{r}^{(n)}\right)$.

Unfortunately, since $d_{i}$ is essentially unknown in practice, $\varsigma_{i}$ is clearly unaccessible. Hence, define $\alpha_{i, j}=\frac{1}{n+2-i-j}$, $l_{i, j} \in \mathbb{R}_{+}, \lambda_{i} \in \mathbb{R}_{+}$which are design parameters, $z_{i, 0}=$ $\hat{x}_{i}, z_{i, 1}=\hat{d}_{i}, z_{i, j}=\widehat{d_{i}^{(j-1)}}$ being the estimation value of $x_{i}, d_{i}$ and $d_{i}^{(j-1)}$ respectively, and $z=\operatorname{col}\left(z_{1,0}, z_{1,2}, \cdots, z_{1, n}, \cdots, z_{n, 1}\right)$. The following HOSMO is constructed in order to estimate the disturbance terms and their corresponding time derivatives [21],

$$
\begin{aligned}
\dot{z}_{i, 0} & =x_{i+1}+f_{i}\left(\bar{x}_{i}\right)+\hbar_{i, 0}, \\
\hbar_{i, 0} & =-l_{i, 0} \lambda_{i}^{\alpha_{i, 0}}\left\lfloor z_{i, 0}-x_{i}\right\rceil^{1-\alpha_{i, 0}}+z_{i, 1}, \\
\hbar_{i, j} & =-l_{i, j} \lambda_{i}^{\alpha_{i, j}}\left\lfloor z_{i, j}-\hbar_{i, j-1}\right\rceil^{1-\alpha_{i, j}}+z_{i, j+1}, j \in \mathbb{N}_{1: n-i}, \\
\hbar_{i, n-i+1} & =-l_{i, n-i+1} \lambda_{i}^{\alpha_{i, n-i+1}}\left\lfloor z_{i, n-i+1}-\hbar_{i, n-i}\right\rceil^{1-\alpha_{i, n-i+1}}, i \in \mathbb{N}_{1: n-1}, \\
\dot{z}_{i, 1} & =\hbar_{i, 1}, \cdots \dot{z}_{i, k}=\hbar_{i, k}, k \in \mathbb{N}_{1: n-i+1}, \\
\dot{z}_{n, 0} & =u+f_{n}(t, x)+\hbar_{n, 0}, \\
\dot{z}_{n, 1} & =\hbar_{n, 1} .
\end{aligned}
$$

Denote $e_{i, 0}=x_{i}-\hat{x}_{i}$ and $e_{i, j}=z_{i, j}-d_{i}^{(j-1)}$. Taking Assumption 2.2 into consideration and combining with (1) and (4), the error dynamics gives as

$$
\begin{aligned}
\dot{e}_{i, 0} & =-l_{i, 0} \lambda_{i}^{\alpha_{i, 0}}\left\lfloor e_{i, 0}\right\rceil^{1-\alpha_{i, 0}}+e_{i, 1}, \\
\dot{e}_{i, j} & =-l_{i, j} \lambda_{i}^{\alpha_{i, j}}\left\lfloor e_{i, j}-\dot{e}_{i, j-1}\right\rceil^{1-\alpha_{i, j}}+e_{i, j+1}, j \in \mathbb{N}_{1: n-i}, \\
\dot{e}_{i, n-i+1} & \in[-N, N] \\
& -l_{i, n-i+1} \lambda_{i}^{\alpha_{i, n-i+1}}\left\lfloor e_{i, n-i+1}-\dot{e}_{i, n-i}\right\rceil^{1-\alpha_{i, n-i+1}} .
\end{aligned}
$$

The following lemma shows that the observer (4) will reconstruct the unknown disturbance signals within a finite time.

Lemma 2 [21] Provided that the observer gain $\lambda_{i}$ is properly selected to satisfy $\lambda_{i}>N, i \in \mathbb{N}_{1: n}$. For any possible well defined trajectory $x(t)$, all states in (5) are uniformly bounded and more specifically, there exists a finite-time $T_{1} \in$ $\mathbb{R}_{+}$such that $e_{i, j}(t)=0, i \in \mathbb{N}_{1: n}, j \in \mathbb{N}_{1: n-i}, \forall t \in\left[T_{1}, \infty\right)$.

With the assistance of the HOSMO (4), by replacing the corresponding term $\frac{\partial d_{i}^{j}}{\partial t^{j}}$ with $z_{i, j+1}$ for $i \in \mathbb{N}_{1: n}, j \in \mathbb{N}_{0: n-i+1}$, now we are able to obtain a series of implementable steadystate reference signals which are accurately generated by the following equations

$x_{i}^{*}=\varsigma_{i}\left(z, \bar{y}_{r}\right), i \in \mathbb{N}_{1: n+1}$.

\subsection{System Transformation}

In order to introduce the non-recursive design framework, a necessary procedure is to transform the given trajectory tracking problem into a stabilization one. To this end, letting

$\eta_{i}=x_{i}-x_{i}^{*}, i \in \mathbb{N}_{1: n}, v=u-x_{n+1}^{*}$,

then the following stabilizable system can be obtained

$\left\{\begin{array}{l}\dot{\eta}_{i}=\eta_{i+1}+f_{i}\left(\bar{x}_{i}\right)-f_{i}\left(\bar{x}_{i}^{*}\right)+\varepsilon_{i}, i \in \mathbb{N}_{1: n-1}, \\ \dot{\eta}_{n}=v+f_{n}(x)-f_{n}\left(x^{*}\right)+\varepsilon_{n}, \\ y_{\eta}=\eta_{1},\end{array}\right.$

where $\bar{x}_{i}^{*}=\operatorname{col}\left(x_{1}^{*}, \cdots, x_{i}^{*}\right), x^{*}=\bar{x}_{n}^{*}, \varepsilon_{i}=f_{i}\left(\bar{x}_{i}^{*}\right)+x_{i+1}^{*}-\dot{x}_{i}^{*}-$ $\left(f_{i}\left(\bar{\zeta}_{i}\right)+\varsigma_{i+1}-\dot{\zeta}_{i}\right), i \in \mathbb{N}_{1: n}$. 
Alternatively, system (8) can also be rewritten as the following compact form

$\dot{\eta}=A \eta+B v+F\left(x, x^{*}\right)+\varepsilon$,

where

$A \triangleq\left[\begin{array}{cccc}0 & 1 & \cdots & 0 \\ \vdots & \vdots & \ddots & \vdots \\ 0 & 0 & \cdots & 1 \\ 0 & 0 & \cdots & 0\end{array}\right], B \triangleq\left[\begin{array}{llll}0 & \cdots & 0 & 1\end{array}\right]^{\top}$

$\eta=\operatorname{col}\left(\eta_{1}, \eta_{2}, \cdots, \eta_{n}\right), F\left(x, x^{*}\right)=\operatorname{col}\left(f_{1}\left(x_{1}\right)-f_{1}\left(x_{1}^{*}\right), \cdots\right.$, $\left.f_{n}(x)-f_{n}\left(x^{*}\right)\right)$ and $\varepsilon=\operatorname{col}\left(\varepsilon_{1}, \cdots, \varepsilon_{n}\right)$.

\subsection{Explicit Controller Construction}

1) Parameter Selection: For system (9), there exists a positive definite and symmetric matrix $P \in \mathbb{R}^{n \times n}$ such that $(A-$ $B K)^{\top} P+P(A-B K) \leq-I_{n}$, where $I_{n}$ is an identity matrix, and $K=\left[k_{1}, k_{2} \cdots, k_{n}\right]$ is a coefficient vector of a Hurwitz polynomial $H(s)=s^{n}+k_{n} s^{n-1}+\cdots+k_{2} s+k_{1}$.

2) Re-Scaling Transformation: Motivated by [20], we define a continuous dynamic gain function $L(t)$ which satisfies $\dot{L} \geq 0, L_{0} \triangleq L(0)=1$. Then, a $\eta-\xi$ system transformation can be depicted as below

$\xi_{i}=\eta_{i} / L^{\varrho+i-1}, i \in \mathbb{N}_{1: n}$,

where $\xi=\operatorname{col}\left(\xi_{1}, \cdots, \xi_{n}\right)$ and the design parameter $\varrho$ subjects to the following guideline:

$\varrho>\max \left\{0,-\frac{\lambda_{\min }(\Theta P+P \Theta)}{2 \lambda_{\min }(P)}\right\}$,

and $\Theta \triangleq \operatorname{diag}[0,1, \cdots, n-1]$.

3) Composite Control Law Construction: At this stage, uniting the disturbance observer (4), a composite controller can be designed for system (1) as the following for

$\left\{\begin{array}{l}v=-L^{\varrho+n} K \xi, u=v+x_{n+1}^{*}, \\ \dot{L}=c \frac{\left(y-y_{r}\right)^{2}}{L^{2 \varrho}}, L_{0}=1\end{array}\right.$

where $c \in \mathbb{R}_{+}$.

Remark 1 In this paper, the proposed synthesis strategy can now be easily implemented without much additional complexities compared with classical recursive design methods, as sketchily depicted by Fig. 1 .

The following 2-D example illustrate the simplicity of the proposed controller design.

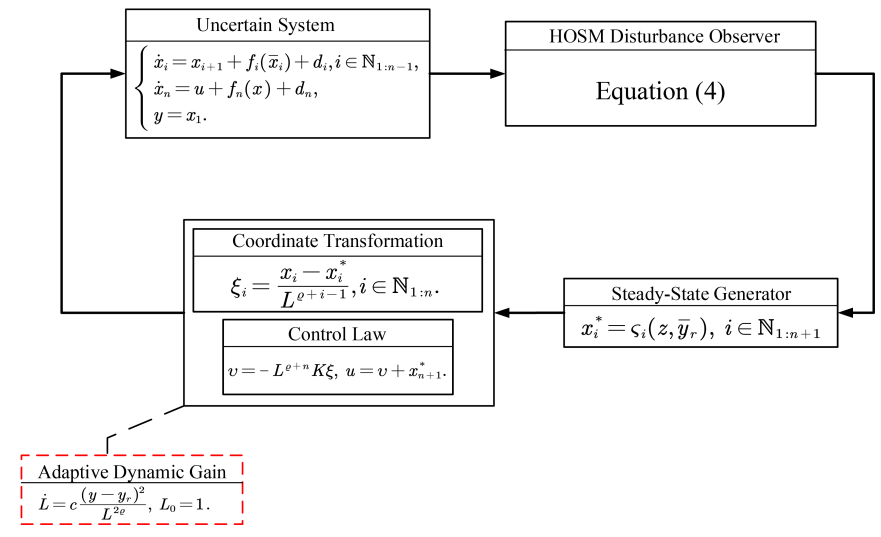

Fig. 1: Block diagram of the proposed control method

Example 1 Consider a system of the following form:

$\left\{\begin{array}{l}\dot{x}_{1}=x_{2}+\sin \left(x_{1}\right)+d_{1}, \\ \dot{x}_{2}=u+\left(x_{2}^{2}+5\right)^{1 / 2}+d_{2}, \\ y=x_{1},\end{array}\right.$

where $d_{1}$ and $d_{2}$ are mismatched or matched disturbances respectively, $y_{r}$ is reference trajectory.

According to the proposed control method, the finitetime disturbance observer can be depicted explicitly as

$\left\{\begin{array}{l}d_{1}\left\{\begin{array}{l}\dot{z}_{1,0}=x_{2}+\sin \left(x_{1}\right)+\hbar_{1,0}, \\ \hbar_{1,0}=-l_{1,0} \lambda_{1}^{1 / 3}\left\lfloor z_{1,0}-x_{1}\right\rceil^{2 / 3}+z_{1,1}, \\ \hbar_{1,1}=-l_{1,1} \lambda_{1}^{1 / 2}\left\lfloor z_{1,1}-\hbar_{1,0}\right\rceil^{1 / 2}+z_{1,2}, \\ \hbar_{1,2}=-l_{1,2} \lambda_{1}\left\lfloor z_{1,2}-\hbar_{1,1}\right\rceil^{0}, \\ \dot{z}_{1, i}=\hbar_{1, i}, i \in \mathbb{N}_{1: 2},\end{array}\right. \\ d_{2}\left\{\begin{array}{l}\dot{z}_{2,0}=u+\left(x_{2}^{2}+5\right)^{1 / 2}+\hbar_{2,0}, \\ \hbar_{2,0}=-l_{2,0} \lambda_{2}^{1 / 2}\left\lfloor z_{2,0}-x_{2}\right\rceil^{1 / 2}+z_{2,1}, \\ \hbar_{2,1}=-l_{2,1} \lambda_{2}\left\lfloor z_{2,1}-\hbar_{2,0}\right\rceil^{0}, \\ \dot{z}_{2,1}=\hbar_{2,1} .\end{array}\right.\end{array}\right.$

The steady-state reference signals can be calculated as: $x_{1}^{*}=y_{r}, x_{2}^{*}=y_{r}^{(1)}-\sin \left(y_{r}\right)-z_{1,1}, x_{3}^{*}=y_{r}^{(2)}-\cos \left(y_{r}\right) y_{r}^{(1)}-$ $z_{1,2}-\left(x_{2}^{* 2}+5\right)^{1 / 2}-z_{2,1}$. The coordinates transformation is expressed as $\xi_{1}=\left(x_{1}-x_{1}^{*}\right) / L^{\varrho}, \xi_{2}=\left(x_{2}-x_{2}^{*}\right) / L^{\varrho+1}$. Therefore, a composite controller can be described as following form

$\dot{L}=c \frac{\left(y-y_{r}\right)^{2}}{L^{2 \varrho}}, L_{0}=1$,

$u=x_{3}^{*}-L^{\varrho+2}\left(k_{1} \xi_{1}+k_{2} \xi_{2}\right)$.

Meanwhile, if apply the method which is based on recursive manner in [14] into system (14), one can get the fol- 
lowing composite controller form:

$$
\begin{aligned}
{\left[\begin{array}{l}
\hat{d}_{1} \\
\hat{d}_{2}
\end{array}\right]=} & {\left[\begin{array}{cc}
\lambda_{1} & 0 \\
0 & \lambda_{2}
\end{array}\right]\left[\begin{array}{l}
x_{1}-p_{1} \\
x_{2}-p_{2}
\end{array}\right], } \\
{\left[\begin{array}{l}
\dot{p_{1}} \\
\dot{p_{2}}
\end{array}\right]=} & {\left[\begin{array}{c}
x_{2} \\
u
\end{array}\right]+\left[\begin{array}{c}
\sin \left(x_{1}\right) \\
\left(x_{2}^{2}+5\right)^{1 / 2}
\end{array}\right]+\left[\begin{array}{l}
\hat{d}_{1} \\
\hat{d}_{2}
\end{array}\right], } \\
x_{2 d}= & -k_{p} \tilde{x}_{1}-\sin \left(x_{1}\right)-\hat{d}_{1}+\dot{x}_{1 d}, \\
u= & -\left(x_{2}^{2}+5\right)^{1 / 2}-\hat{d}_{2}-\left(k_{p}+\cos \left(x_{1}\right)\right)\left(x_{2}+\sin \left(x_{1}\right)+\hat{d}_{1}\right) \\
& +k_{p} \dot{x}_{1 d}+\ddot{x}_{1 d}-k_{q} \tilde{x_{2}},
\end{aligned}
$$

where $\lambda_{1}, \lambda_{2}>0, k_{p}, k_{q}>0$ are design parameters and $p_{1}, p_{2}$ are auxiliary state variables, respectively, $x_{1 d}$ denotes a reference signal and $\tilde{x}_{1}=x_{1}-x_{1 d}, \tilde{x}_{2}=x_{2}-x_{2 d}$ represent the tracking errors.

As one can observe from (15) and (16) that the proposed controller form is much simpler than the controller derived from backstepping method in [14], which could largely facilitate the practical implementations. Second-order system could reflect the complexity of recursive method, and it is conceivable that as the increasing of system order, the controller form will be increasingly redundant. Meanwhile, different with recursive methods, even if the nonlinearity term $f_{i}(\cdot)$ changes, the proposed controller form is still applicable, which greatly improves the portability of the proposed controller design framework.

\subsection{Stability Analysis}

In this section, we will carry out rigorous proofs of stability via the proposed composite controller of the form (13).

Keeping (11) and (13) in mind, system (8) can be compactly expressed as the following form

$\dot{\xi}=L(A-B K) \xi-\left(\varrho I_{n}+\Theta\right) \frac{\dot{L}}{L} \xi+\tilde{F}\left(L, \varrho, x, x^{*}\right)+\tilde{\varepsilon}$,

where $\tilde{F}\left(L, \varrho, x, x^{*}\right)=\operatorname{col}\left(\left(f_{1}\left(x_{1}\right)-f_{1}\left(x_{1}^{*}\right)\right) / L^{\varrho}, \cdots,\left(f_{n}(x)-\right.\right.$ $\left.\left.f_{n}\left(x^{*}\right)\right) / L^{\varrho+n-1}\right)$ and $\tilde{\varepsilon}=\operatorname{col}\left(\varepsilon_{1} / L^{\varrho}, \cdots, \varepsilon_{n} / L^{\varrho+n-1}\right)$.

Theorem 1 Considering the closed-loop system (1)-(4)-(13) under Assumption 2.2, the following statements hold:

1) All the signals in the closed-loop system are globally uniformly bounded.

2) $\lim _{t \rightarrow \infty} y=y_{r}$.

proof: Define a Lyapunov candidate as $V(\xi)=\xi^{\top} P \xi$. Taking the time derivative of $V(\xi)$ along system $(17), \dot{V}(\xi)$ can be depicted as

$$
\begin{aligned}
\dot{V}(\xi)= & \frac{\partial V(\xi)}{\partial \xi^{\top}} L(A-B K) \xi-\frac{\partial V(\xi)}{\partial \xi^{\top}}\left(\varrho I_{n}+\Theta\right) \frac{\dot{L}}{L} \xi \\
& +\frac{\partial V(\xi)}{\partial \xi^{\top}} \tilde{F}\left(L, \varrho, x, x^{*}\right)+\frac{\partial V(\xi)}{\partial \xi^{\top}} \tilde{\varepsilon} .
\end{aligned}
$$

In what follows, some necessary steps are presented for rigourous stability analysis.

Firstly, keeping the parameter matrix $P$ in mind, it yields naturally that

$\frac{\partial V(\xi)}{\partial \xi^{\top}} L(A-B K) \xi \leq-L\|\xi\|^{2}$

Secondly, according to above guideline of $\varrho, 2 \varrho P+\Theta P+$ $P \Theta$ can be rendered as positive definite, that is, $\left(2 \varrho \lambda_{\min }(P)+\right.$ $\left.\lambda_{\min }(\Theta P+P \Theta)\right)>0$. Taking $\dot{L} / L \geq 0$ into consideration, we have:

$$
\begin{aligned}
\frac{\partial V(\xi)}{\partial \xi^{\top}}\left(\varrho I_{n}+\Theta\right) \frac{\dot{L}}{L} \xi & \geq\left(2 \varrho \lambda_{\min }(P)+\lambda_{\min }(\Theta P+P \Theta)\right) \frac{\dot{L}}{L}\|\xi\|^{2} \\
& =\kappa_{1} \cdot \frac{\dot{L}}{L}\|\xi\|^{2} \geq 0
\end{aligned}
$$

where $\kappa_{1}=\left(2 \varrho \lambda_{\min }(P)+\lambda_{\min }(\Theta P+P \Theta)\right)$.

Thirdly, recalling that the nonlinear function terms in system (1) satisfy the Lipschitz continuous condition, the following relation holds:

$$
\begin{aligned}
\frac{f_{i}\left(\bar{x}_{i}\right)-f_{i}\left(\bar{x}_{i}^{*}\right)}{L^{\varrho+i-1}} & =\frac{\mu_{i}}{L^{\varrho+i-1}}\left(\left|x_{1}-x_{1}^{*}\right|+\left|x_{2}-x_{2}^{*}\right|+\cdots\left|x_{i}-x_{i}^{*}\right|\right) \\
& =\frac{\mu_{i}}{L^{\varrho+i-1}}\left(L^{\varrho}\left|\xi_{1}\right|+L^{\varrho+1}\left|\xi_{2}\right| \cdots+L^{\varrho+i-1}\left|\xi_{i}\right|\right) \\
& \leq \mu_{i}\left(\left|\xi_{1}\right|+\left|\xi_{2}\right|+\cdots+\left|\xi_{i}\right|\right) \leq \mu_{i} \cdot \sqrt{n}\|\xi\|
\end{aligned}
$$

where $\mu_{i}$ is a Lipschitz constant related to $f(\cdot)$. Then we get

$$
\begin{aligned}
\frac{\partial V(\xi)}{\partial \xi^{\top}} \tilde{F}\left(L, \varrho, x, x^{*}\right) & \leq \sum_{i=1}^{n} \frac{\partial V(\xi)}{\partial \xi_{i}} \cdot \frac{f_{i}\left(\bar{x}_{i}\right)-f_{i}\left(\bar{x}_{i}^{*}\right)}{L^{\varrho+i-1}} \\
& \leq \sum_{i=1}^{n} \sqrt{n} \mu_{i}\|\xi\| \cdot \frac{\partial V(\xi)}{\partial \xi_{i}} \\
& \leq \sqrt{n} \mu\|\xi\| \cdot\left\|\frac{\partial V(\xi)}{\partial \xi}\right\| \\
& \leq 2 n \mu \lambda_{\max }(P)\|\xi\|^{2}=\kappa_{2}\|\xi\|^{2}
\end{aligned}
$$

where $\mu=\mu_{i \max }, \kappa_{2}=2 n \mu \lambda_{\max }(P)$.

Finally, if $x(t)$ is well defined, it is evident that the value of $\tilde{\varepsilon}$ is related to the convergence time $T_{1}$ of finite-time disturbance observer, so the stability analysis of $\tilde{\varepsilon}$ will be divided into two sections, i.e., $t \in\left[0, T_{1}\right]$ and $t \in\left[T_{1}, \infty\right]$.

In the case of $0<t<T_{1}$, it follows straightforwardly from Lemma 2 that there must exist a bounded constant $E \in$ $\mathbb{R}_{+}$such that $\sup \left\{\left|\varepsilon_{i}\right|\right\} \leq E$. By applying Lemma 1 , we arrive at $i \in \mathbb{N}_{1: n}$

$$
\begin{aligned}
\frac{\partial V(\xi)}{\partial \xi^{\top}} \tilde{\varepsilon} & \leq 2 \lambda_{\max }(P)\|\xi\| \cdot\|\tilde{\varepsilon}\| \leq 2 \sqrt{n} \lambda_{\max }(P)\|\xi\| E \\
& \leq \kappa_{3}\|\xi\|^{2}+E^{2}
\end{aligned}
$$

where $\kappa_{3}$ is a positive constant. 
Then combining with (19)-(23), (18) can be rewritten as

$$
\begin{aligned}
\dot{V}(\xi) & \leq-\left(L-\kappa_{2}-\kappa_{3}\right)\|\xi\|^{2}-\kappa_{1} \cdot \frac{\dot{L}}{L}\|\xi\|^{2}+E^{2} \\
& \leq-\left(L-\kappa_{2}-\kappa_{3}\right)\|\xi\|^{2}+E^{2} .
\end{aligned}
$$

In the case of $t \geq T_{1}$, the following relations hold: $e_{i, j}=$ $0 \Rightarrow d_{i}^{j}=\hat{d}_{i}^{j} \Rightarrow x_{i}^{*}=\varsigma_{i} \Rightarrow \varepsilon_{i}=0$. Substituting (19)-(22) into (18), the time derivative of $V(\xi)$ can be described as the following form

$\dot{V}(\xi) \leq-\left(L-\kappa_{2}\right)\|\xi\|^{2}$.

The following properties can be concluded with a simple deduction, that are, starting from any initial condition $\xi(0) \in \mathbb{R}^{n}$ and $L_{0}=1$, the solution $(\xi(t), L(t))$ of the closedloop system (13)-(17) existing on $[0,+\infty)$ is bounded, meanwhile, $\lim _{t \rightarrow \infty} \xi(t)=0, \lim _{t \rightarrow \infty} L=\bar{L} \in R_{+}$.

Subsequently, the boundness of dynamic gain $L$ will be claimed. Firstly, one can observe that $L$ can not escape at $t=t_{f}$, and here this paper only consider the case $T_{1} \leq t_{f}$. To better illustrate this case, suppose that $\lim _{t \rightarrow t_{f}} L=+\infty$. Since $\dot{L} \geq 0, L(t)$ is monotone non-decreasing. Hence there exists a finite time $t_{1} \in\left[0, T_{1}\right)$ which could make the following relationships hold:

$L \geq \kappa_{2}+\kappa_{3}+1, t \in\left[t_{1}, \infty\right)$

that is,

$\dot{V}(\xi) \leq-\|\xi\|^{2}+E^{2}, t \in\left[t_{1}, T_{1}\right)$,

$\dot{V}(\xi) \leq-\|\xi\|^{2}, t \in\left[T_{1}, t_{f}\right)$.

As is known that, $\int_{t_{1}}^{t_{f}} c \xi_{1}(t)^{2} d t \leq \int_{t_{1}}^{t_{f}} c\|\xi(t)\|^{2} d t$. Then, we arrive at

$$
\begin{aligned}
+\infty & =L\left(t_{f}\right)-L\left(t_{1}\right)=\int_{t_{1}}^{t_{f}} \dot{L} d t=\int_{t_{1}}^{t_{f}} c \xi_{1}(t)^{2} d t \\
& \leq c \int_{t_{1}}^{t_{f}}\|\xi(t)\|^{2} d t=c\left(\int_{t_{1}}^{T_{1}}\|\xi(t)\|^{2} d t+\int_{T_{1}}^{t_{f}}\|\xi(t)\|^{2} d t\right) \\
& \leq c\left[V\left(\xi\left(t_{1}\right)\right)-V\left(\xi\left(t_{f}\right)\right)+E^{2}\left(T_{1}-t_{1}\right)\right]=\text { constant }
\end{aligned}
$$

it is clear that the inequality leads to a contradiction. Hence, the dynamic gain $L$ is well defined and bounded on $\left[0, t_{f}\right)$.

Finally, we present that $\xi$ is bounded on interval $\left[0, t_{f}\right)$. To this end, taking the Lyapunov function into consideration firstly, a direct computation gives

$$
V(\xi(t))-V(\xi(0)) \geq \lambda_{\min }(P)\|\xi\|^{2}-\xi(0)^{\top} P \xi(0),
$$

then the following change of coordinates is introduced:

$\Xi_{i}=\frac{\eta_{i}}{L^{*} \varrho+i-1}, i=1,2, \cdots, n$,

where $L^{*}$ is a constant which satisfies

$L^{*} \geq \max \left\{L\left(t_{f}\right), \kappa_{2}+\kappa_{3}+1\right\}$, then the system can be transformed into

$$
\begin{aligned}
& \dot{\Xi}_{1}=L^{*} \Xi_{2}+\frac{F_{1}\left(\bar{x}_{1}\right)}{L^{* \varrho}}+\frac{\varepsilon_{1}}{L^{* \varrho}}, \\
& \dot{\Xi}_{2}=L^{*} \Xi_{3}+\frac{F_{2}\left(\bar{x}_{2}\right)}{L^{*} \varrho+1}+\frac{\varepsilon_{2}}{L^{*} \varrho+1} \\
& \vdots \\
& \dot{\Xi}_{n}=\frac{v^{*}}{L^{*} \varrho+n-1}+\frac{F_{n}\left(\bar{x}_{n}\right)}{L^{*} \varrho+n-1}+\frac{\varepsilon_{n}}{L^{*} \varrho+n-1}
\end{aligned}
$$

where $F_{i}=f_{i}\left(\bar{x}_{i}\right)-f_{i}\left(\bar{x}_{i}^{*}\right)$. The following compact form can be written along above equation

$\dot{\Xi}=L^{*} A \Xi+\frac{1}{L^{*} \varrho+n-1} B v^{*}+\tilde{F}+\tilde{\varepsilon}$,

that is

$\dot{\Xi}=L^{*}(A-B K) \Xi+\tilde{F}+\tilde{\varepsilon}$,

where $v^{*}=\frac{-K E}{L^{*} \varrho+n}, \tilde{F}=\operatorname{col}\left(\left(f_{1}\left(x_{1}\right)-f_{1}\left(x_{1}^{*}\right)\right) / L^{* \varrho}, \cdots,\left(f_{n}(x)-\right.\right.$ $\left.\left.f_{n}\left(x^{*}\right)\right) / L^{* \varrho+n-1}\right)$ and $\tilde{\varepsilon}=\operatorname{col}\left(\varepsilon_{1} / L^{* \varrho}, \cdots, \varepsilon_{n} / L^{* \varrho+n-1}\right)$.

Now taking the Lyapunov function as $V_{2}=\Xi^{\top} P \Xi$ for system (28) into consideration, a straightforward calculation shows that along the trajectories of (28), the following holds

$$
\begin{aligned}
\dot{V}_{2}(\Xi(t)) & \leq-L^{*}\|\Xi\|^{2}+\kappa_{2}\|\Xi\|^{2}+\kappa_{3}\|\Xi\|^{2}+E^{2} \\
& =-\left(L^{*}-\kappa_{2}-\kappa_{3}\right)\|\Xi\|^{2}+E^{2},
\end{aligned}
$$

then, substituting (29) into (26), one get

$$
\begin{array}{r}
\lambda_{\min }(P)\|\Xi\|^{2}-\Xi^{\top}(0) P \Xi(0) \leq V_{2}(\Xi(t))-V_{2}(\Xi(0)) \\
\leq-\left(L^{*}-\kappa_{2}-\kappa_{3}\right) \int_{0}^{t}\|\Xi\|^{2} d t+E^{2} t
\end{array}
$$

that is,

$$
\begin{gathered}
\lambda_{\min }(P)\|\Xi\|^{2} \leq E^{2} t+\Xi^{\top}(0) P \Xi(0), \\
\int_{0}^{t}\|\Xi\|^{2} d t \leq E^{2} t+\Xi^{\top}(0) P \Xi(0) .
\end{gathered}
$$

Since $L$ is bounded on $\left[0, t_{f}\right)$, it is concluded immediately from (31) that $\Xi$ and $\int_{o}^{t} \Xi d t$ are bounded on $\left[0, t_{f}\right)$. In view of (11) and (27), it results in the boundedness of $\int_{0}^{t}\|\xi\|^{2} d t$ and $\xi$ on $\left[0, t_{f}\right)$. Meanwhile, the conclusion can be also obtained when $t_{1} \in\left[T_{1}, t_{f}\right)$ and the process of proof is similar to that of $t_{1} \in\left[0, T_{1}\right)$. Hence it can be straightforwardly deduced from Barbalat's Lemma that $\lim _{t \rightarrow \infty} \xi(t)=0$ will arrive to $\lim _{t \rightarrow \infty} x(t)=x^{*}(t)$, which represents the proposed method can realize the exact tracking control objective that is, $\lim _{t \rightarrow \infty} y=$ $y_{r}$.

This completes the proof of Theorem 1 . 


\section{Application to a single-link robot arm system}

A single-link robot arm system is modeled as following form [23]:

$$
\begin{aligned}
\dot{v}_{1} & =v_{2}, \\
\dot{v}_{2} & =\frac{K}{J_{2} N} v_{3}-\frac{F_{2}}{J_{2}} v_{2}-\frac{K}{J_{2}} v_{1}-\frac{m g d}{J_{2}} \cos v_{1}+d_{2}, \\
\dot{v}_{3} & =v_{4}, \\
\dot{v}_{4} & =\frac{1}{J_{1}} u+\frac{K}{J_{1} N} v_{1}-\frac{K}{J_{2} N} v_{3}-\frac{F_{1}}{J_{1}} v_{4}+d_{4}, \\
y & =v_{1},
\end{aligned}
$$

where $J_{1}, J_{2}, K, N, m, g, d$ are given parameters, while $F_{1}$ and $F_{2}$ are viscous friction coefficients. $d_{2}, d_{4}$ are lump disturbances including external disturbances and internal uncertainties. The control objective is to regulate the robotic system to the equilibrium $\left(v_{1}, v_{2}, v_{3}, v_{4}, u\right)=(0,0, \operatorname{mgdN} / K, 0$, $m g J_{1} / J_{2}$ ).

By introducing the coordinates transformation, the system can be transformed into following form,

$$
\begin{aligned}
& x_{1}=v_{1}, x_{2}=v_{2}, x_{3}=\frac{K}{J_{2} N} v_{3}-\frac{m g d}{J_{2}}, x_{4}=\frac{K}{J_{2} N} v_{4}, \\
& v=\frac{K}{J_{2} N}\left(\frac{u}{J_{1}}-\frac{m g d}{J_{2}}\right) .
\end{aligned}
$$

Then we arrive at

$$
\begin{aligned}
& \dot{x}_{1}=x_{2}, \\
& \dot{x}_{2}=x_{3}-\frac{F_{2}}{J_{2}} x_{2}-\frac{K}{J_{2}} x_{1}-\frac{m g d}{J_{2}}\left(\cos \left(x_{1}\right)-1\right)+d_{2}, \\
& \dot{x}_{3}=x_{4} \\
& \dot{x}_{4}=v+\frac{K^{2}}{J_{1} J_{2} N^{2}} x_{1}-\frac{K}{J_{2} N} x_{3}-\frac{F_{1}}{J_{1}} x_{4}+d_{4}, \\
& y=x_{1} .
\end{aligned}
$$

Parameters in the simulation are set as follows: $K / J_{2}=$ $5, m g d / J_{2}=4, K^{2} /\left(J_{1} J_{2} N^{2}\right)=2, K /\left(J_{2} N\right)=3, \frac{F_{2}}{J_{2}}=6, \frac{F_{1}}{J_{1}}=$ 4. The finite-time disturbance observer can be depicted explicitly as

$$
\left\{\begin{array}{l}
d_{2}\left\{\begin{array}{l}
\dot{z}_{2,0}=x_{3}-6 x_{2}-5 x_{1}-4\left(\cos \left(x_{1}\right)-1\right)+\hbar_{2,0}, \\
\hbar_{2,0}=-l_{2,0} \lambda_{2}^{1 / 4}\left\lfloor z_{2,0}-x_{2}\right\rceil^{3 / 4}+z_{2,1}, \\
\hbar_{2,1}=-l_{2,1} \lambda_{2}^{1 / 3}\left\lfloor z_{2,1}-\hbar_{2,0}\right\rceil^{2 / 3}+z_{2,2}, \\
\hbar_{2,2}=-l_{2,2} \lambda_{2}^{1 / 2}\left\lfloor z_{2,2}-\hbar_{2,1}\right\rceil^{1 / 2}+z_{2,3}, \\
\hbar_{2,3}=-l_{2,3} \lambda_{2}\left\lfloor z_{2,3}-\hbar_{2,2}\right\rceil^{0} \\
\dot{z}_{2, i}=\hbar_{2, i}, i \in \mathbb{N}_{1: 3},
\end{array}\right. \\
d_{4}\left\{\begin{array}{l}
\dot{z}_{4,0}=u+2 x_{1}-3 x_{3}-4 x_{4}+\hbar_{4,0}, \\
\hbar_{4,0}=-l_{4,0} \lambda_{4}^{1 / 2}\left\lfloor z_{4,0}-x_{4}\right\rceil^{1 / 2}+z_{4,1}, \\
\hbar_{4,1}=-l_{4,1} \lambda_{4}\left\lfloor z_{4,1}-\hbar_{4,0}\right\rceil^{0} \\
\dot{z}_{4,1}=\hbar_{4,1},
\end{array}\right.
\end{array}\right.
$$

where the observer gains are chosen as $\left(l_{2,0}, l_{2,1} l_{2,2}, l_{2,3}, \lambda_{2}\right)=$ $(20,20,30,20,1),\left(l_{4,0}, l_{4,1}, \lambda_{4}\right)=(20,20,1)$.
In the following simulation, assume $d_{2}(t)=2 a \sin (t)$, $d_{4}(t)=a \cos (2 t), a \in \mathbb{R}_{+}$, and the initial condition is set as $\left(x_{1}(0), x_{2}(0), x_{3}(0), x_{4}(0)\right)=(-9,-1,4,2)$. The steady-state reference signals can be calculated as: $y_{r}=0, x_{1}^{*}=y_{r}, x_{2}^{*}=$ $y_{r}^{(1)}, x_{3}^{*}=y_{r}^{(2)}+6 y_{r}^{(1)}+5 y_{r}+4\left(\cos \left(y_{r}\right)-1\right)-z_{2,1}, x_{4}^{*}=y_{r}^{(3)}+$ $6 y_{r}^{(2)}+5 y_{r}^{(1)}-4 \sin \left(y_{r}\right) y_{r}^{(1)}-z_{2,2}, x_{5}^{*}=-2 y_{r}+3\left(y_{r}^{(2)}+6 y_{r}^{(1)}+5 y_{r}+\right.$ $\left.4\left(\cos \left(y_{r}\right)-1\right)-z_{2,1}\right)+4\left(y_{r}^{(3)}+6 y_{r}^{(2)}+5 y_{r}^{(1)}-4 \sin \left(y_{r}\right) y_{r}^{(1)}-z_{2,2}-\right.$ $z_{4,1}+y_{r}^{(4)}+6 y_{r}^{(3)}+5 y_{r}^{(2)}-z_{2,3}-4 \sin \left(y_{r}\right) y_{r}^{(2)}-4 \cos \left(y_{r}\right)\left(y_{r}^{(1)}\right)^{2}$. The coordinates transformation is expressed as $\xi_{1}=\left(x_{1}-\right.$ $\left.x_{1}^{*}\right) / L^{\varrho}, \xi_{2}=\left(x_{2}-x_{2}^{*}\right) / L^{\varrho+1}, \xi_{3}=\left(x_{3}-x_{3}^{*}\right) / L^{\varrho+2}, \xi_{4}=$ $\left(x_{4}-x_{4}^{*}\right) / L^{\varrho+3}$.

Then the composite controller can be described as following simple form

$$
\begin{aligned}
& \dot{L}=c \frac{\left(y-y_{r}\right)^{2}}{L^{2 \varrho}}, L_{0}=1, \\
& u=x_{5}^{*}-L^{\varrho+4}\left(k_{1} \xi_{1}+k_{2} \xi_{2}+k_{3} \xi_{3}+k_{4} \xi_{4}\right) .
\end{aligned}
$$

In this case, the parameters of the adaptive updated law and the controller are chosen as $\left(k_{1}, k_{2}, k_{3}, k_{4}, c, \varrho\right)=(0.8,0.01$, $0.2,0.01,0.14,2.5)$, and if we use robust control strategy, $L$ will be determined by a guideline in [16]. Hence, $L$ is chosen as 2.5 in the simulation.

The result of the simulation is presented in Fig. 2. As one can clearly observe that the proposed update mechanism of $L$ can adaptively adjust, in order to handle with different levels of disturbances. Meanwhile, if the case is that when $L$ is a fixed constant (e.g., the worst case design), the convergence rate is slower than the proposed method. Further, as the increasing of $L$, the transient-time performance of robust control usually has bigger overshoot, which is caused by the robustness redundancy of the closed-loop system and could cause some side effects for the controlled system.

\section{Conclusion}

In this paper, we propose an nonrecursive controller to realize exact tracking control goal. Compared with related results, several distinguishable improvements can be obtained. Firstly, the update mechanism could adaptively handle various working conditions. Secondly, the proposed controller is portable for different nonlinearity. Moreover, the proposed one-step control design will lead to a concise controller form, which largely facilitate the practical implementations.

\section{References}

1. S. Li, J. Yang, W. Chen and X. Chen, Disturbance Observer-Based Control: Methods and Applications, Boca Raton, FL, USA: CRC Press, 2014.

2. W. Chen, "Nonlinear disturbance observer-enhanced dynamic inversion control of missiles," Journal of Guidance, Control, and Dynamics. 26(1), 161-166, (2003). 

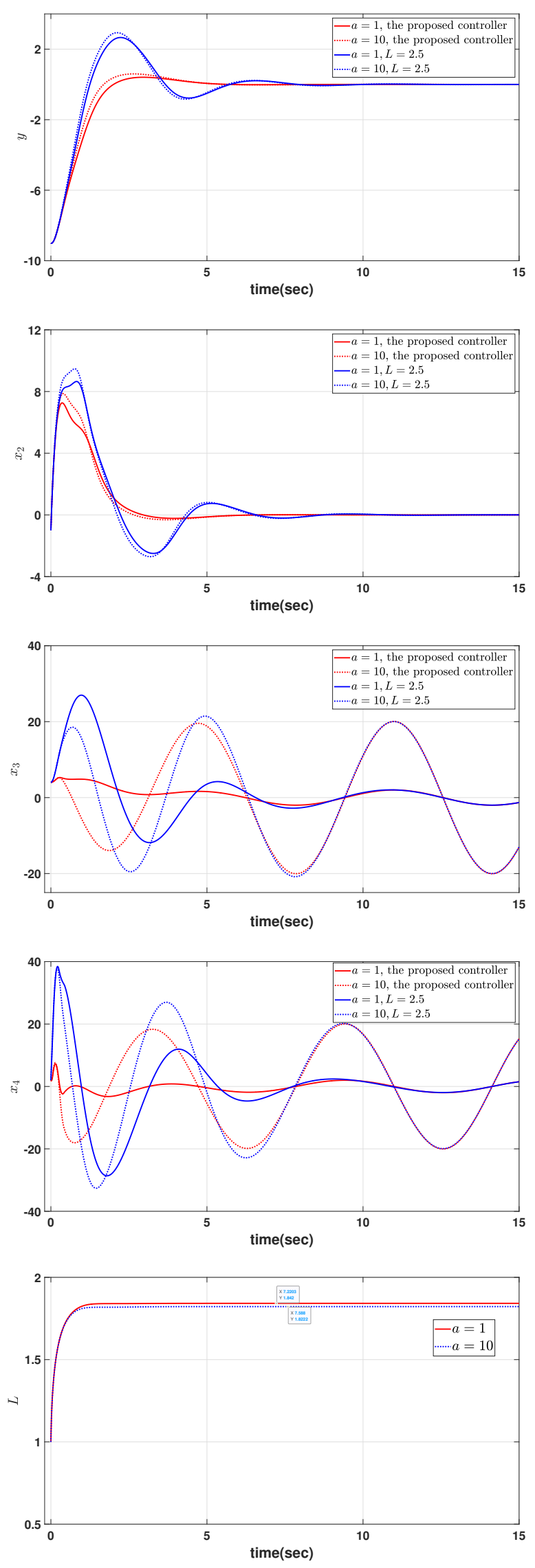

Fig. 2: The transient-response of closed-loop in single-link robot arm system
3. J. Han, "From PID to active disturbance rejection control," IEEE Transactions on Industrial Electronics. 56(3), 900-906, (2009).

4. B. Leonid and H. K. Khalil, "Performance recovery of feedbacklinearization-based designs," IEEE Transactions on Automatic Control. 53(10), 2324-2334, (2008).

5. Y. Liu and Y. Jing, "Practical finite-time almost disturbance decoupling strategy for uncertain nonlinear systems," Nonlinear Dyn. 95, 117-128, (2019).

6. X. Li, S. Zhang, C. Zhang, Y. Zhou and C. Zhang, "An improved deadbeat predictive current control scheme for open-winding permanent magnet synchronous motors drives with disturbance observer," IEEE Transactions on Power Electronics. 36(4), 46224632, (2021).

7. Y. Zhu, J. Qiao and L. Guo, "Adaptive sliding mode disturbance observer-based composite control with prescribed performance of space manipulators for target capturing," IEEE Transactions on Industrial Electronics. 66(3), 1973-1983, (2019).

8. G. V. Lakhekar, L. M. Waghmare and R. G. Roy, "Disturbance observer-based fuzzy adapted s-surface controller for spatial trajectory tracking of autonomous underwater vehicle," IEEE Transactions on Intelligent Vehicles. 4(4), 622-636, (2019).

9. L. Qu, W. Qiao and L. Qu, "Active-disturbance-rejection-based sliding-mode current control for permanent-magnet synchronous motors," IEEE Transactions on Power Electronics. 36(1), 751-760, (2021).

10. R. Errouissi, A. Al-Durra and M. Debouza, "A novel design of PI current controller for PMSG-based wind turbine considering transient performance specifications and control saturation," IEEE Transactions on Industrial Electronics. 65(11), 8624-8634, (2018).

11. S. Hwang and H. S. Kim, "Extended disturbance observer-based integral sliding mode control for nonlinear system via T-S fuzzy model," IEEE Access. 8, 116090-116105, (2020).

12. C. Zhang, J. Yang and S. Li, "A generalized active disturbance rejection control method for nonlinear uncertain systems subject to additive disturbance," Nonlinear Dyn. 83, 2361-2372, (2015).

13. J, Yang, S. Li and X. Yu, "Sliding-mode control for systems with mismatched uncertainties via a disturbance observer," IEEE Transaction on Industrial Electronics. 60(1), 160-169, (2013).

14. H. Sun, S. Li, J. Yang and L. Guo, "Non-linear disturbance observer-based back-stepping control for airbreathing hypersonic vehicles with mismatched disturbances," IET Control Theory and Applications. 8(17), 1852-1865, (2014).

15. A. Estrada and L. Fridman, "Quasi-continuous HOSM control for systems with unmatched perturbations," Automatica. 46(11), 19161919, (2010).

16. C. Zhang, Y. Yan, C. Wen, J. Yang and H. Yu, "A nonsmooth composite control design framework for nonlinear systems with mismatched disturbances: Algorithms and experimental tests," IEEE Transactions on Industrial Electronics. 65 (11), 8828-8839, (2018).

17. H. Lei and W. Lin, "Universal adaptive control of nonlinear systems with unknown growth rate by output feedback," Automatica. 42(10), 1783-1789, (2006).

18. P. Krishnamurthy and F. Khorrami, "Dynamic high-gain scaling: state and output feedback with application to systems with iss appended dynamic driven by all states," IEEE Transactions on Automatic Control. 49(12), 2219-2239, (2004).

19. M. Koo, H. Choi and J. Lim, "Universal control of nonlinear systems with unknown nonlinearity and growth rate by adaptive output feedback," Automatica. 47(10), 2211-2217, (2011).

20. Y. Yan, C. Zhang, A. Narayan, J. Yang, S. Li and H. Yu, "Generalized dynamic predictive control for nonparametric uncertain systems with application to series elastic actuators," IEEE Transactions on Industrial Informatics. 14(11), 4829-4840, (2018).

21. A. Levant, "Higher-order sliding modes: differentiation and output-feedback control," International Journal of Control. 76(9), 924-941, (2003). 
22. H. K. Khalil, Nonlinear Systems, New York: Macmillan Publishing Company, (1992).

23. A. Isidori, Nonlinear Control System, New York: Springer, (1995). 
Figures

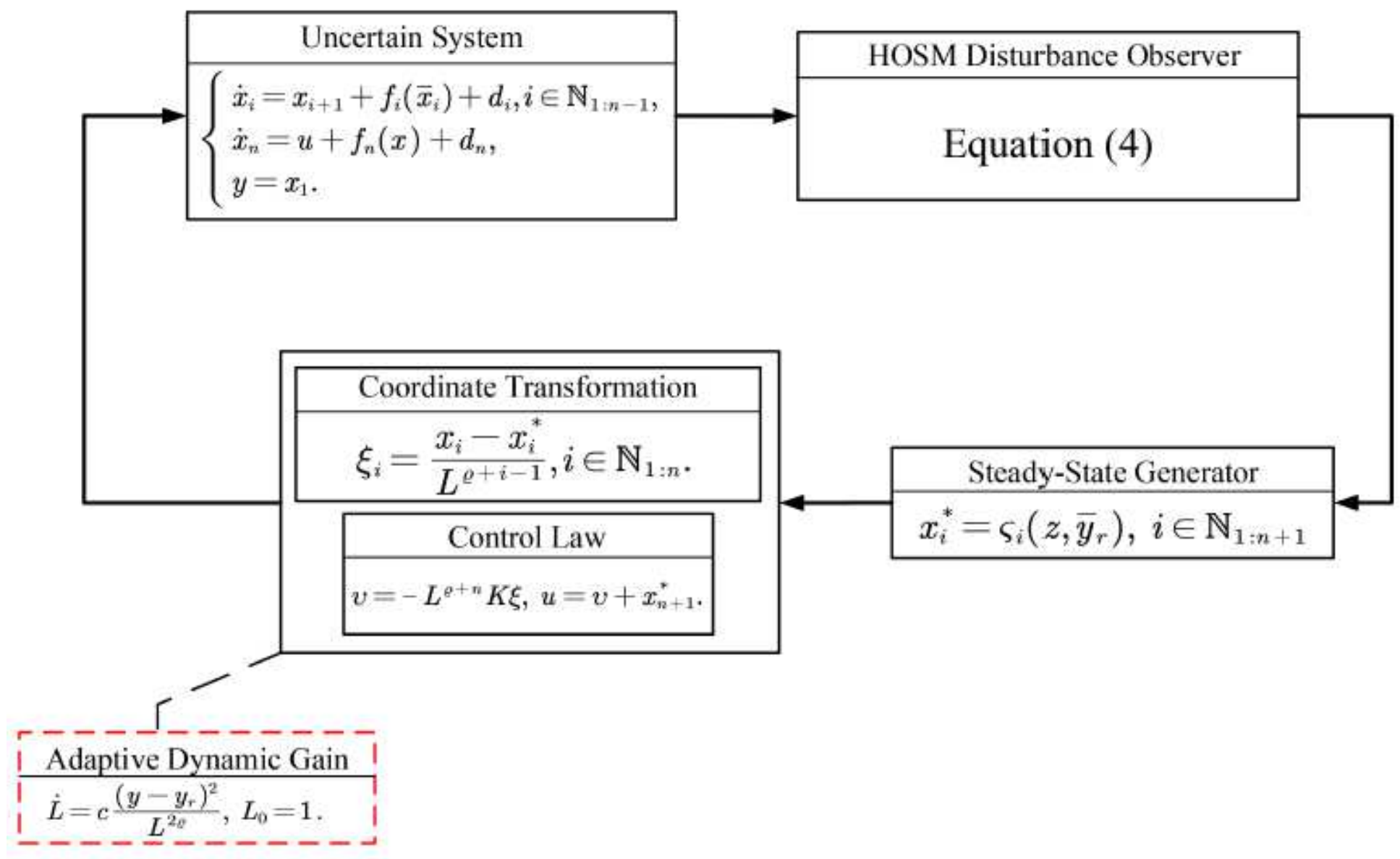

\section{Figure 1}

Block diagram of the proposed control method 

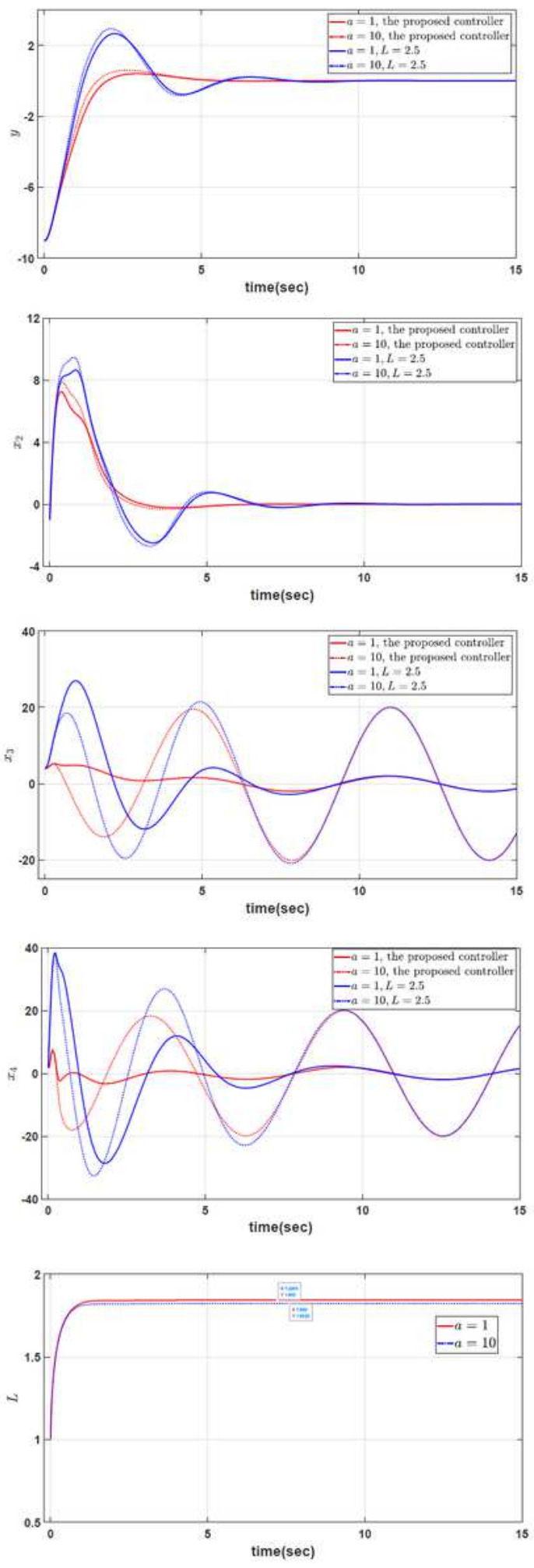

Figure 2

The transient-response of closed-loop in single-link robot arm system 